\title{
HAUSDORFF DIMENSION OF LIMSUP SETS OF RECTANGLES IN THE HEISENBERG GROUP
}

\author{
FREDRIK EKSTRÖM ${ }^{1}$, ESA JÄRVENPÄ̈̈${ }^{2}$ and MAARIT JÄRVENPÄ̈̈ ${ }^{3}$
}

\begin{abstract}
The almost sure value of the Hausdorff dimension of limsup sets generated by randomly distributed rectangles in the Heisenberg group is computed in terms of directed singular value functions.
\end{abstract}

\section{Introduction}

Dimensional properties of subsets of Heisenberg groups have attained a lot of interest recently. Due to the non-trivial relation between the Hausdorff dimensions with respect to the Euclidean and the Heisenberg metrics [4, 5], one cannot directly transfer dimensional results in Euclidean spaces into Heisenberg groups. Indeed, it turns out that some theorems concerning dimensions have a special flavour or even an essentially different form in the Heisenberg setting. These include, for example, dimensional properties of self-affine sets, projections and slices.

In the Heisenberg group Hausdorff dimensions of self-similar and self-affine sets have been studied e.g. in $[1,5,6,11]$. Even though the class of affine iterated function systems is quite restrictive - every such system is a horizontal lift of an affine iterated function system on the plane - the dimension calculations involve some subtleties. The behaviour of the Hausdorff dimension under projections and slicing transpires to be interesting, see $[2,3,19,21]$. There are two kinds of natural projections (and slices) in Heisenberg groups - the horizontal and vertical ones. The vertical projections possess an exceptional feature: they are not Lipschitz continuous. This indicates that the methods developed in the Euclidean setting cannot be utilised. For related questions concerning Sobolev maps and the foliations generated by the horizontal subspaces, see [7].

In this paper, we initiate a new direction of research in Heisenberg groups by investigating dimensions of limsup sets generated by rectangles. Let $X$ be a space and let $\left(A_{n}\right)$ be a sequence of subsets of $X$. The limsup set generated by

2010 Mathematics Subject Classification. 28A80, 60D05. 
the sequence $\left(A_{n}\right)$ consists of those points of $X$ which are covered by infinitely many of the sets $A_{n}$, that is,

$$
\underset{n}{\limsup } A_{n}=\bigcap_{n=1}^{\infty} \bigcup_{k=n}^{\infty} A_{k} .
$$

Limsup sets are encountered in many fields of mathematics - one of the earliest appearances being the Borel-Cantelli lemma [9, 10]. They play a central role in the study of Besicovitch-Eggleston sets concerning the $k$-adic expansions of real numbers $[8,13]$ as well as in Diophantine approximation [22, 25]. For more information on different aspects of limsup sets, we refer to [20] and the references therein. For recent results regarding Diophantine approximation in Heisenberg groups, see [30, 31, 32].

Dimensional properties of random limsup sets have been actively studied, see for example $[12,15,17,18,20,23,24,26,27,29]$. Combining the results of these papers, the almost sure value of dimension of random limsup sets is known in the following cases:

- the underlying space $X$ is a Riemann manifold, the generating sets $\left(A_{n}\right)$ are Lebesgue measurable with positive density and the driving measure determining the randomness is not singular with respect to the Lebesgue measure,

- $X$ is the Euclidean or the symbolic space, the generating sets $\left(A_{n}\right)$ are balls and the driving measure has special properties like being a Gibbs measure,

- $X$ is an Ahlfors regular metric space, randomness is given by the natural measure and $\left(A_{n}\right)$ is a sequence of balls.

In [14] a dimension formula for limsup sets generated by rectangles in products of Ahlfors regular metric spaces is derived. In this paper, we address the problem of determining the Hausdorff dimension of random limsup sets generated by rectangles in the first Heisenberg group (see Theorem 1.1 below). In [14] the Lipschitz continuity of projections is utilised to a great extent, and because of that, the same methods cannot be used in our setting. Instead, we will extend some results known in the Euclidean setting to unimodular groups or to compact metric spaces, and make calculations specific to the Heisenberg group to complete the argument.

We proceed by introducing our notation. The Heisenberg group $\mathbb{H}$ is the set $\mathbb{R}^{3}$ with the non-commutative group operation

$$
p p^{\prime}=\left(x+x^{\prime}, y+y^{\prime}, z+z^{\prime}+2\left(x y^{\prime}-y x^{\prime}\right)\right)
$$


where $p=(x, y, z)$ and $p^{\prime}=\left(x^{\prime}, y^{\prime}, z^{\prime}\right)$. The unit in $\mathbb{H}$ is $(0,0,0)$ and the inverse of $p$ is $p^{-1}=(-x,-y,-z)$. There is a norm on $\mathbb{H}$ given by

$$
\|p\|=\left(\left(x^{2}+y^{2}\right)^{2}+z^{2}\right)^{1 / 4}
$$

which gives rise to a left-invariant metric

$$
d_{\mathbb{H}}\left(p, p^{\prime}\right)=\left\|p^{-1} p^{\prime}\right\|=\left(\left(\left(x^{\prime}-x\right)^{2}+\left(y^{\prime}-y\right)^{2}\right)^{2}+\left(z^{\prime}-z-2\left(x y^{\prime}-y x^{\prime}\right)\right)^{2}\right)^{1 / 4} .
$$

Both left and right translation in $\mathbb{H}$ move vertical lines to vertical lines in such a way that the Euclidean distance between lines is preserved, and the image of the Lebesgue measure on a vertical line under translation is the Lebesgue measure on the image line. Thus Fubini's theorem implies that the Lebesgue measure on $\mathbb{R}^{3}$ is invariant under translations in $\mathbb{H}$. It is easy to see that the Lebesgue measure of $B(0, r)$ in $\mathbb{H}$ is proportional to $r^{4}$, and by translation invariance the same is true for every ball of radius $r$. In particular, the metric space $\left(\mathbb{H}, d_{\mathbb{H}}\right)$ has Hausdorff dimension 4.

Let

$$
L(0)=\left\{p^{\prime} ; x^{\prime}=y^{\prime}=0\right\} \text { and } H(0)=\left\{p^{\prime} ; z^{\prime}=0\right\}
$$

be the vertical line and horizontal plane through the origin, respectively. For $p \in \mathbb{H}$, define

$$
L(p)=p L(0) \text { and } H(p)=p H(0) .
$$

Then $L(p)$ is the vertical line through $p$ and

$$
H(p)=\left\{p^{\prime} ; z^{\prime}=z+2\left(x y^{\prime}-y x^{\prime}\right)\right\}
$$

is the plane through $p$ that has slope 0 in the direction $(x, y)$ and slope $2\left(x^{2}+y^{2}\right)^{1 / 2}$ in the orthogonal direction $(-y, x)$. Note that

$$
d_{\mathbb{H}}\left(p, p^{\prime}\right) \geq\left(\left(x^{\prime}-x\right)^{2}+\left(y^{\prime}-y\right)^{2}\right)^{1 / 2},
$$

with equality if and only if $p^{\prime} \in H(p)$. It follows that the distance to $L\left(p^{\prime}\right)$ from any point on $L(p)$ equals the Euclidean distance from $(x, y)$ to $\left(x^{\prime}, y^{\prime}\right)$, and vice versa by symmetry. Thus vertical lines are parallel in the Heisenberg metric, and the distance between them is the same as the Euclidean distance. The symmetry of the metric implies that $p^{\prime} \in H(p)$ if and only if $p \in H\left(p^{\prime}\right)$ and, by definition, $p H\left(p^{\prime}\right)=H\left(p p^{\prime}\right)$. If $p^{\prime} \in L(p)$ then $H\left(p^{\prime}\right)$ is parallel in the Euclidean sense to $H(p)$.

A closed rectangle in $\mathbb{H}$ centred at 0 is

$$
\bar{R}(0, r)=\left\{p^{\prime} ; x^{\prime 2}+y^{\prime 2} \leq r_{1}^{2},\left|z^{\prime}\right| \leq r_{2}^{2}\right\},
$$

and, in general, a closed rectangle centred at $p$ is a set of the form

$\bar{R}(p, r)=p \bar{R}(0, r)=\left\{p^{\prime} ;\left(x^{\prime}-x\right)^{2}+\left(y^{\prime}-y\right)^{2} \leq r_{1}^{2},\left|z^{\prime}-z-2\left(x y^{\prime}-y x^{\prime}\right)\right| \leq r_{2}^{2}\right\}$, 
where $r=\left(r_{1}, r_{2}\right)$. This is the set of points that can be reached from $p$ by moving "horizontally" in $H(p)$ a distance at most $r_{1}$ and then vertically a distance at most $r_{2}$, or by moving first vertically a distance at most $r_{2}$ and then horizontally a distance at most $r_{1}$.

Let $p=\left(p_{n}\right)$ be a sequence of points in $\mathbb{H}$ and let $\underline{r}=\left(r_{n}\right)$ be a bounded sequence of pairs of positive numbers, and define

$$
E_{\underline{r}}(\underline{p})=\limsup _{n} \bar{R}\left(p_{n}, r_{n}\right) .
$$

The purpose of this article is to give a formula for the Hausdorff dimension of such a set when the centres of the rectangles are chosen randomly. Let $\lambda$ be the Lebesgue measure on $\mathbb{H}$ and let $W$ be a bounded open subset of $\mathbb{H}$. Let $\lambda_{W}=\left.\lambda(W)^{-1} \lambda\right|_{W}$ and define the probability space $(\Omega, \mathbf{P})$ by $\Omega=\mathbb{H}^{\mathbb{N}}$ and $\mathbf{P}=\lambda_{W}^{\mathbb{N}}$. Then $\omega \mapsto E_{\underline{r}}(\omega)$ can be considered as a random set defined on $(\Omega, \mathbf{P})$. The directed singular value function is defined as follows: for $r=\left(r_{1}, r_{2}\right)$, if $r_{1} \leq r_{2}$ let

$$
\Phi^{t}(r)= \begin{cases}r_{2}^{t} & \text { if } t \in[0,2] \\ r_{1}^{t-2} r_{2}^{2} & \text { if } t \in[2,4]\end{cases}
$$

and if $r_{1} \geq r_{2}$ let

$$
\Phi^{t}(r)= \begin{cases}r_{1}^{t} & \text { if } t \in[0,3] \\ r_{1}^{6-t} r_{2}^{2(t-3)} & \text { if } t \in[3,4] .\end{cases}
$$

The minimum of numbers $a, b \in \mathbb{R}$ is denoted by $a \wedge b$.

THEOREM 1.1. With the above notation,

$$
\operatorname{dim}_{\mathrm{H}} E_{\underline{r}}=\inf \left\{t ; \sum_{n} \Phi^{t}\left(r_{n}\right)<\infty\right\} \wedge 4
$$

$\mathbf{P}$-almost surely.

Let $X$ be a metric space. The $t$-dimensional Hausdorff content of a subset $A$ of $X$ is defined by

$$
\mathcal{H}_{\infty}^{t}(A)=\left\{\sum_{n}\left|C_{n}\right|^{t} ;\left\{C_{n}\right\} \text { is a cover of } A\right\},
$$

where the diameter of a set $C$ is denoted by $|C|$. If $\mu$ is a Borel measure on $X$ and $t>0$, the $t$-energy of $\mu$ is defined by

$$
I_{t}(\mu)=\iint d(x, y)^{-t} \mathrm{~d} \mu(y) \mathrm{d} \mu(x) .
$$


The $t$-capacity of a Borel subset $A$ of $X$ is defined by

$$
\operatorname{Cap}_{t}(A)=\sup \left\{\frac{1}{I_{t}(\mu)} ; \mu \in \mathcal{P}(A)\right\},
$$

where $\mathcal{P}(A)$ denotes the set of Borel probability measures on $X$ that give full measure to $A$. It can be shown that

$$
\operatorname{Cap}_{t}(A) \leq \mathcal{H}_{\infty}^{t}(A)
$$

always holds (see [20, Remark 3.3]).

The proof of Theorem 1.1 is based on estimating the Hausdorff content and capacity of rectangles in the Heisenberg group. The following proposition is proved in Section 4.

Proposition 1.2. For all $t \in(0,4) \backslash\{1,2,3\}$, there is a constant $c_{t}$ such that

$$
c_{t}^{-1} \mathcal{H}_{\infty}^{t}(\bar{R}(x, r)) \leq \Phi^{t}(r) \leq c_{t} \operatorname{Cap}_{t}(\bar{R}(x, r))
$$

for every $x$ and $r$.

This immediately implies that if $\sum_{n} \Phi^{t}\left(r_{n}\right)<\infty$ then $\mathcal{H}^{t}\left(E_{\underline{r}}(\omega)\right)=0$ for every $\omega$, since every tail of the sequence of rectangles is a cover of $E_{\underline{r}}(\omega)$. The almost sure lower bound for $\operatorname{dim}_{H} E_{\underline{r}}$ then follows from the estimate of the capacity of a rectangle together with Theorem 1.3 below.

A Borel measure $\mu$ on a metric space $X$ is $(c, d)$-regular if

$$
c^{-1} r^{d} \leq \mu(B(x, r)) \leq c r^{d}
$$

for every $x \in X$ and $r \in[0,|X|]$. A locally compact group $G$ is unimodular if the left invariant Haar measure is also right invariant, or equivalently, if it is invariant under inversion. For example, the Heisenberg group is $(c, 4)$-regular for some $c$ and unimodular.

Theorem 1.3 is a variant of $[20$, Theorem $1.1(\mathrm{~b})]$ and $[26$, Theorem 1]. We give a somewhat different proof even though the main philosophy follows the same lines as the proofs in $[20,26]$.

THEOREM 1.3. Let $G$ be a unimodular group with left invariant metric and $(c, d)$-regular Haar measure $\lambda$, and let $W$ be a bounded open subset of $G$ such that $\lambda(W)=1$. Define the probability space $(\Omega, \mathbf{P})$ by $\Omega=G^{\mathbb{N}}$ and $\mathbf{P}=\left(\left.\lambda\right|_{W}\right)^{\mathbb{N}}$. Let $\left(V_{n}\right)$ be a bounded sequence of open subsets of $G$ (bounded meaning that there is a ball in $G$ that contains $V_{n}$ for every $\left.n\right)$. For $\omega=\left(\omega_{n}\right) \in \Omega$ let

$$
E(\omega)=\lim \sup \left(\omega_{n} V_{n}\right) .
$$

If $t \in(0, d)$ and

$$
\sum_{n} \operatorname{Cap}_{t}\left(V_{n}\right)=\infty
$$


then there exists $g \in G$ so that almost every $\omega$ is such that $\mathcal{H}^{t}(\mathcal{U} \cap E(\omega))=\infty$ for every open subset $\mathcal{U}$ of $W g$.

The assumption that $\lambda(W)=1$ makes the proof slightly easier to read, but is not essential since a constant multiple of a $(c, d)$-regular Haar measure is a $\left(c^{\prime}, d\right)$-regular Haar measure for some $c^{\prime}$.

The proof of Theorem 1.3 is based on the following deterministic lemma (compare [16, Lemma 7], [20, Proposition 4.6] and [28, Theorem 1]), which is proved in Section 2. If $X$ is a locally compact metric space, the weak-* topology on the space of Radon measures on $X$ is the topology generated by the maps $\{\mu \mapsto \mu(\varphi)\}$, where $\varphi$ ranges over the continuous compactly supported functions $X \rightarrow \mathbb{R}$. It is not difficult to see that $\lim _{n \rightarrow \infty} \mu_{n}=\mu$ in the weak-* topology if and only if $\lim _{n \rightarrow \infty} \mu_{n}(\varphi)=\mu(\varphi)$ for every continuous compactly supported function $\varphi$. Naturally, this also defines the weak-* topology in the case $X$ is compact.

Lemma 1.4. Let $\nu$ be a finite Borel measure on a compact metric space $X$ and let $\left(\varphi_{n}\right)_{n=1}^{\infty}$ be a sequence of non-negative continuous functions on $X$ such that $\lim _{n \rightarrow \infty} \varphi_{n} \mathrm{~d} \nu=\nu$ in the weak-* topology and $\liminf _{n \rightarrow \infty} I_{t}\left(\varphi_{n} \rho \mathrm{d} \nu\right) \leq I_{t}(\rho \mathrm{d} \nu)$ whenever $\rho$ is a product of finitely many of the functions $\left\{\varphi_{n}\right\}$. Then for every $t>0$,

$$
\operatorname{Cap}_{t}\left(\operatorname{supp} \nu \cap \limsup _{n}\left(\operatorname{supp} \varphi_{n}\right)\right) \geq \frac{\nu(X)^{2}}{I_{t}(\nu)}
$$

Notation and conventions

All measures appearing below are Radon measures, but this will not be explicitly stated. Thus "measure" below means "Radon measure". If $X$ is a metric space with a measure $\mu$ and $\varphi$ is a non-negative continuous function on $X$, then $I_{t}(\varphi)$ means $I_{t}(\varphi \mathrm{d} \mu)$. Similarly, if $A$ is a Borel subset of $X$ then $I_{t}(A)$ means $I_{t}\left(\left.\mu\right|_{A}\right)$. If $X$ is a locally compact metric space, then the space of Radon measures on $X$ is considered as a topological space under the weak-* topology.

\section{Proof of Lemma 1.4}

Let $Y$ be a topological space. A function $f: Y \rightarrow(-\infty, \infty]$ is lower semicontinuous if $f^{-1}((a, \infty])$ is open for every $a \in \mathbb{R}$, or equivalently if $f\left(y_{0}\right) \leq$ $\liminf _{y \rightarrow y_{0}} f(y)$ for every $y_{0} \in Y$. The following lemma is well known, but a proof is included for the convenience of the reader.

Lemma 2.1. Let $X$ be a compact metric space and let $t>0$. Then $\mu \mapsto I_{t}(\mu)$ is lower semicontinuous. 
Proof. Let $\mathcal{M}(X)$ be the space of finite measures on $X$. It will first be shown that the map $\mathcal{M}(X) \rightarrow \mathcal{M}(X) \times \mathcal{M}(X), \mu \mapsto \mu \times \mu$ is continuous. Let $\eta$ be a continuous function on $X \times X$ and let $\mu_{0} \in \mathcal{M}(X)$. It suffices to show that for every $\varepsilon>0$, the set

$$
A=\left\{\mu \in \mathcal{M}(X) ;\left|(\mu \times \mu)(\eta)-\left(\mu_{0} \times \mu_{0}\right)(\eta)\right|<3 \varepsilon\right\}
$$

contains an open neighbourhood of $\mu_{0}$.

Let $K>\mu_{0}(X)$. By Stone-Weierstrass' theorem, there are functions $\left\{\eta_{i}\right\}_{i=1}^{n}$ of the form $\eta_{i}=\eta_{i, 1} \times \eta_{i, 2}$, where $\eta_{i, j}$ are continuous, such that $\left\|\eta-\sum_{i=1}^{n} \eta_{i}\right\|_{\infty}<$ $\varepsilon / K^{2}$. The set

$V=\left\{\mu \in \mathcal{M}(X) ;\left|(\mu \times \mu)\left(\eta_{i}\right)-\left(\mu_{0} \times \mu_{0}\right)\left(\eta_{i}\right)\right|<\frac{\varepsilon}{n}\right.$ for every $i$ and $\left.\mu(X)<K\right\}$ is open since $(\mu \times \mu)\left(\eta_{i}\right)=\mu\left(\eta_{i, 1}\right) \mu\left(\eta_{i, 2}\right)$, and $\mu_{0} \in V$. If $\mu \in V$ then

$$
\begin{aligned}
\left|(\mu \times \mu)(\eta)-\left(\mu_{0} \times \mu_{0}\right)(\eta)\right| & \leq\left|(\mu \times \mu)(\eta)-(\mu \times \mu)\left(\sum_{i=1}^{n} \eta_{i}\right)\right| \\
& +\sum_{i=1}^{n}\left|(\mu \times \mu)\left(\eta_{i}\right)-\left(\mu_{0} \times \mu_{0}\right)\left(\eta_{i}\right)\right| \\
& +\left|\left(\mu_{0} \times \mu_{0}\right)\left(\sum_{i=1}^{n} \eta_{i}\right)-\left(\mu_{0} \times \mu_{0}\right)(\eta)\right|<3 \varepsilon,
\end{aligned}
$$

so that $V$ is a subset of $A$.

Define

$$
I_{t}^{M}(\mu)=\iint d(x, y)^{-t} \wedge M \mathrm{~d} \mu(y) \mathrm{d} \mu(x) .
$$

Let $a \in \mathbb{R}$ and let $\mu_{0}$ be a measure in $\mathcal{M}(X)$ such that $I_{t}\left(\mu_{0}\right)>a$. Then there exists $M$ such that $I_{t}^{M}\left(\mu_{0}\right)>a$, and the set $\left\{\mu ; I_{t}^{M}(\mu)>a\right\}$ is open, contains $\mu_{0}$ and is contained in $\left\{\mu ; I_{t}(\mu)>a\right\}$.

Proof of Lemma 1.4. Let $\varepsilon \in(0, \nu(X))$ and let $\left(\varepsilon_{k}\right)$ be a sequence of positive numbers such that $\sum_{k} \varepsilon_{k} \leq \varepsilon$. Define recursively a sequence $\left(n_{k}\right)_{k=1}^{\infty}$ of natural numbers as follows, using the notation $\rho_{k}=\varphi_{n_{k}} \cdot \ldots \cdot \varphi_{n_{1}}$ with the convention $\rho_{0}=1$. For $k \geq 1$, assume that $n_{1}, \ldots, n_{k-1}$ are defined. Then since $\lim _{n \rightarrow \infty} \nu\left(\varphi_{n} \rho_{k-1}\right)=\nu\left(\rho_{k-1}\right)$ and

$$
\liminf _{n \rightarrow \infty} I_{t}\left(\varphi_{n} \rho_{k-1}\right) \leq I_{t}\left(\rho_{k-1}\right),
$$

it is possible to find $n_{k}>n_{k-1}$ such that

$$
\nu\left(\rho_{k}\right) \geq \nu\left(\rho_{k-1}\right)-\varepsilon_{k}
$$


and

$$
I_{t}\left(\rho_{k}\right) \leq I_{t}\left(\rho_{k-1}\right)+\varepsilon_{k}
$$

Let $\mu$ be an accumulation point of the sequence of measures $\left(\rho_{k} \mathrm{~d} \nu\right)$ - then there is a strictly increasing sequence $\left(k_{i}\right)$ such that $\mu=\lim _{i \rightarrow \infty} \rho_{k_{i}} \mathrm{~d} \nu$. Thus

$$
\mu(X)=\lim _{i \rightarrow \infty} \nu\left(\rho_{k_{i}}\right) \geq \liminf _{k \rightarrow \infty} \nu\left(\rho_{k}\right) \geq \nu(X)-\sum_{k} \varepsilon_{k} \geq \nu(X)-\varepsilon
$$

and by Lemma 2.1,

$$
I_{t}(\mu) \leq \liminf _{i \rightarrow \infty} I_{t}\left(\rho_{k_{i}}\right) \leq \limsup _{k \rightarrow \infty} I_{t}\left(\rho_{k}\right) \leq I_{t}(\nu)+\sum_{k} \varepsilon_{k} \leq I_{t}(\nu)+\varepsilon .
$$

Moreover,

$$
\operatorname{supp} \mu \subset \operatorname{supp} \nu \cap \bigcap_{k} \operatorname{supp} \varphi_{n_{k}} \subset \operatorname{supp} \nu \cap \limsup _{n}\left(\operatorname{supp} \varphi_{n}\right)
$$

and thus

$$
\operatorname{Cap}_{t}\left(\operatorname{supp} \nu \cap \limsup _{n}\left(\operatorname{supp} \varphi_{n}\right)\right) \geq \frac{\mu(X)^{2}}{I_{t}(\mu)} \geq \frac{(\nu(X)-\varepsilon)^{2}}{I_{t}(\nu)+\varepsilon} .
$$

Letting $\varepsilon \rightarrow 0$ concludes the proof.

\section{Proof of Theorem 1.3}

The proof of Theorem 1.3 is based on constructing a sequence $\left(\varphi_{n}^{\omega}\right)$ of random continuous functions supported on a compact neighbourhood of $W$, satisfying the hypothesis of Lemma 1.4, such that

$$
\limsup _{n}\left(\operatorname{supp} \varphi_{n}^{\omega}\right) \subset \underset{n}{\lim \sup }\left(\omega_{n} V_{n}\right) .
$$

A few lemmas are needed. The first one is used in the proofs of Lemma 3.2 and Theorem 1.3.

Lemma 3.1. Let $(X, \lambda)$ be a $(c, d)$-regular space and let $t \in(0, d)$. Then there is a constant $C$ such that for every $x \in X$ and $r>0$,

$$
I_{t}(B(x, r)) \leq C r^{2 d-t}
$$

Proof. If $\varphi$ is a non-negative Borel function on $X$ then

$$
\int \varphi \mathrm{d} \lambda=\int_{0}^{\infty} \lambda\{z \in X ; \varphi(z)>\gamma\} \mathrm{d} \gamma
$$


since both sides equal the $\lambda \times \mathcal{L} e b$-measure of the set $\{(z, u) \in X \times[0, \infty] ; u \in(0, \varphi(z))\}$ (note that the boundary of this set has measure 0 ). Thus for $y \in X$,

$$
\begin{aligned}
\int_{B(x, r)} d(y, z)^{-t} \mathrm{~d} \lambda(z)=\int_{0}^{\infty} \lambda\left\{z \in B(x, r) ; d(y, z)^{-t}>\gamma\right\} \mathrm{d} \gamma \\
=\int_{0}^{\infty} \lambda\left(B(x, r) \cap B\left(y, \gamma^{-1 / t}\right)\right) \mathrm{d} \gamma \leq c \int_{0}^{\infty} \min \left(r^{d}, \gamma^{-d / t}\right) \mathrm{d} \gamma \\
=c \int_{0}^{r^{-t}} r^{d} \mathrm{~d} \gamma+c \int_{r^{-t}}^{\infty} \gamma^{-d / t} \mathrm{~d} \gamma=\frac{c d}{d-t} r^{d-t},
\end{aligned}
$$

and thus

$$
I_{t}(B(x, r)) \leq \lambda(B(x, r)) \cdot \frac{c d}{d-t} r^{d-t} \leq \frac{c^{2} d}{d-t} r^{2 d-t}
$$

The next lemma is a variant of [20, Proposition 3.8].

Lemma 3.2. Let $(X, \lambda)$ be a locally compact $(c, d)$-regular space and let $\theta$ be a finite compactly supported measure on $X$. Then there is a sequence $\left(\varphi_{n}\right)$ of nonnegative continuous compactly supported functions on $X$ such that $\lim _{n \rightarrow \infty} \varphi_{n} \mathrm{~d} \lambda=$ $\theta$ (in the weak-* topology) and $\limsup _{n \rightarrow \infty} I_{t}\left(\varphi_{n}\right) \leq I_{t}(\theta)$ for every $t \in(0, d)$.

Proof. For each $n$, let $\left\{x_{n, i}\right\}$ be a maximal (finite or countable) $1 / n$-separated subset of $X$. Then $\left\{B\left(x_{n, i}, 1 / 2 n\right)\right\}$ are disjoint and $\left\{B\left(x_{n, i}, 1 / n\right)\right\}$ is a cover of $X$. Let $Q_{n, i}$ be the set of points $x$ in $X$ for which $i$ is the first index such that $d\left(x, x_{n, i}\right)=\min _{j} d\left(x, x_{n, j}\right)$, that is,

$$
\begin{gathered}
Q_{n, i}=\left\{x \in X ; d\left(x, x_{n, i}\right)<d\left(x, x_{n, j}\right) \text { for } j=1, \ldots, i-1\right. \text { and } \\
\left.d\left(x, x_{n, i}\right) \leq d\left(x, x_{n, j}\right) \text { for } j=i, \ldots, N_{n}\right\} .
\end{gathered}
$$

Then, for each $n,\left\{Q_{n, i}\right\}$ is a partition of $X$ into Borel sets and

$$
B\left(x_{n, i}, \frac{1}{2 n}\right) \subset Q_{n, i} \subset B\left(x_{n, i}, \frac{1}{n}\right)
$$

for every $i$.

For each $i$, let

$$
\varphi_{n, i}(x)=a_{n, i} \max \left(0,1-4 n d\left(x, x_{n, i}\right)\right),
$$

where $a_{n, i}$ is such that $\lambda\left(\varphi_{n, i}\right)=1$. Then $\varphi_{n, i}$ is supported on $\bar{B}\left(x_{n, i}, 1 / 4 n\right)$, and $a_{n, i} \leq 2^{1+3 d} c n^{d}$ since

$$
\int_{B\left(x_{n, i}, \frac{1}{4 n}\right)}\left(1-4 n d\left(x, x_{n, i}\right)\right) \mathrm{d} \lambda(x) \geq \frac{1}{2} \lambda\left(B\left(x_{n, i}, \frac{1}{8 n}\right)\right) \geq 2^{-(1+3 d)} c^{-1} n^{-d} .
$$


Let

$$
\varphi_{n}=\sum_{i} \theta\left(Q_{n, i}\right) \varphi_{n, i} .
$$

Note that, for every $x$, there are only finitely many non-zero terms in the sum defining $\varphi_{n}$. Further, $\varphi_{n}$ is compactly supported since $\theta$ has a compact support.

Let $\eta$ be a continuous compactly supported function on $X$ and let $\varepsilon>0$. Then $\eta$ is uniformly continuous and there is some $n_{0}$ such that

$$
\left|\eta(x)-\eta\left(x_{n, i}\right)\right| \leq \frac{\varepsilon}{2 \theta(X)}
$$

whenever $n \geq n_{0}$ and $x \in Q_{n, i}$. Then for $n \geq n_{0}$,

$$
\begin{aligned}
& \left|\theta(\eta)-\left(\varphi_{n} \mathrm{~d} \lambda\right)(\eta)\right| \leq \\
& \quad \sum_{i}\left(\int_{Q_{n, i}}\left|\eta-\eta\left(x_{n, i}\right)\right| \mathrm{d} \theta+\theta\left(Q_{n, i}\right) \int_{Q_{n, i}}\left|\eta\left(x_{n, i}\right)-\eta\right| \varphi_{n, i} \mathrm{~d} \lambda\right) \leq \varepsilon
\end{aligned}
$$

using that $\left(\varphi_{n, i} \mathrm{~d} \lambda\right)\left(Q_{n, i}\right)=1$ for every $i$. Thus $\lim _{n \rightarrow \infty} \varphi_{n} \mathrm{~d} \lambda=\theta$. It remains to show that $\limsup _{n \rightarrow \infty} I_{t}\left(\varphi_{n}\right) \leq I_{t}(\theta)$, and for this it may be assumed that $I_{t}(\theta)<\infty$.

Let $n$ be a natural number and $\alpha>1$, and if $\psi_{1}, \psi_{2}$ are continuous compactly supported functions on $X$ let

$$
J_{t}\left(\psi_{1}, \psi_{2}\right)=\iint d(x, y)^{-t} \psi_{1}(x) \psi_{2}(y) \mathrm{d} \lambda(x) \mathrm{d} \lambda(y) .
$$

Then

$$
I_{t}\left(\varphi_{n}\right)=S_{1}+S_{2}+S_{3}
$$

where

$$
\begin{aligned}
& S_{1}=\sum_{d\left(x_{n, i}, x_{n, j}\right) \geq \frac{\alpha}{n}} \theta\left(Q_{n, i}\right) \theta\left(Q_{n, j}\right) J_{t}\left(\varphi_{n, i}, \varphi_{n, j}\right), \\
& S_{2}=\sum_{\substack{d\left(x_{n, i}, x_{n, j}\right)<\frac{\alpha}{n} \\
i \neq j}} \theta\left(Q_{n, i}\right) \theta\left(Q_{n, j}\right) J_{t}\left(\varphi_{n, i}, \varphi_{n, j}\right), \\
& S_{3}=\sum_{i} \theta\left(Q_{n, i}\right)^{2} I_{t}\left(\varphi_{n, i}\right) .
\end{aligned}
$$

If $x \in \operatorname{supp} \varphi_{n, i}$ and $y \in \operatorname{supp} \varphi_{n, j}$ and $d\left(x_{n, i}, x_{n, j}\right) \geq \alpha / n$, then

$$
\left|d(x, y)-d\left(x_{n, i}, x_{n, j}\right)\right| \leq \frac{1}{n} \leq \frac{d\left(x_{n, i}, x_{n, j}\right)}{\alpha},
$$


so that

Thus for $i, j$ appearing in $S_{1}$,

$$
\frac{\alpha-1}{\alpha} \leq \frac{d(x, y)}{d\left(x_{n, i}, x_{n, j}\right)} \leq \frac{\alpha+1}{\alpha} .
$$

$$
\begin{aligned}
\theta\left(Q_{n, i}\right) \theta\left(Q_{n, j}\right) J_{t}\left(\varphi_{n, i}, \varphi_{n, j}\right) & \leq\left(\frac{\alpha}{\alpha-1}\right)^{t} \theta\left(Q_{n, i}\right) \theta\left(Q_{n, j}\right) d\left(x_{n, i}, x_{n, j}\right)^{-t} \\
& \leq\left(\frac{\alpha+1}{\alpha-1}\right)^{t} \iint_{Q_{n, i} \times Q_{n, j}} d(x, y)^{-t} \mathrm{~d} \theta(x) \mathrm{d} \theta(y),
\end{aligned}
$$

and it follows that

$$
S_{1} \leq\left(\frac{\alpha+1}{\alpha-1}\right)^{t} I_{t}(\theta) .
$$

If $x \in \operatorname{supp} \varphi_{n, i}$ and $y \in \operatorname{supp} \varphi_{n, j}$ then $i \neq j$ implies that $d(x, y) \geq 1 / 2 n$ and if $x \in Q_{n, i}$ and $y \in Q_{n, j}$ then $d\left(x_{n, i}, x_{n, j}\right) \leq \alpha / n$ implies that $d(x, y) \leq(\alpha+2) / n$. Thus

$$
\begin{aligned}
S_{2} & \leq 2^{t} \sum_{\substack{d\left(x_{n, i}, x_{n, j}\right)<\frac{\alpha}{n} \\
i \neq j}} \theta\left(Q_{n, i}\right) \theta\left(Q_{n, j}\right) n^{t} \\
& \leq 2^{t}(\alpha+2)^{t} \sum_{d\left(x_{n, i}, x_{n, j}\right)<\frac{\alpha}{n}} \iint_{Q_{n, i} \times Q_{n, j}} d(x, y)^{-t} \mathrm{~d} \theta(x) \mathrm{d} \theta(y) \\
& \leq 2^{t}(\alpha+2)^{t} \iint_{d(x, y) \leq \frac{\alpha+2}{n}} d(x, y)^{-t} \mathrm{~d} \theta(x) \mathrm{d} \theta(y) .
\end{aligned}
$$

By Lemma 3.1 there is a constant $\mathrm{C}$ such that

$$
I_{t}(B(x, r)) \leq C r^{2 d-t}
$$

for every $x \in X$ and $r>0$. It follows that

$$
I_{t}\left(\varphi_{n, i}\right) \leq a_{n, i}^{2} I_{t}\left(B\left(x_{n, i}, \frac{1}{4 n}\right)\right) \leq C^{\prime} n^{t},
$$

where $C^{\prime}=4^{1+3 d} c^{2} C$, so that

$$
\begin{aligned}
S_{3} & \leq C^{\prime} \sum_{i} \theta\left(Q_{n, i}\right)^{2} n^{t} \leq 2^{t} C^{\prime} \sum_{i} \iint_{Q_{n, i} \times Q_{n, i}} d(x, y)^{-t} \mathrm{~d} \theta(x) \mathrm{d} \theta(y) \\
& \leq 2^{t} C^{\prime} \iint_{d(x, y) \leq \frac{2}{n}} d(x, y)^{-t} \mathrm{~d} \theta(x) \mathrm{d} \theta(y) .
\end{aligned}
$$

Given $\varepsilon>0$ it is possible to choose $\alpha$ large enough so that $S_{1} \leq I_{t}(\theta)+\varepsilon$, and then $n_{0}$ large enough so that $S_{2}+S_{3} \leq \varepsilon$ for every $n \geq n_{0}$. It follows that $\limsup _{n \rightarrow \infty} I_{t}\left(\varphi_{n}\right) \leq I_{t}(\theta)+2 \varepsilon$, and letting $\varepsilon \rightarrow 0$ concludes the proof. 
The following lemma is a modification of the argument from [20, p. 1580].

Lemma 3.3. Let $(X, \lambda)$ be a complete $(c, d)$-regular space and let $\left(V_{n}\right)$ be a sequence of open subsets of an open ball $B$ in $X$ such that $\sum_{n} \operatorname{Cap}_{t}\left(V_{n}\right)=\infty$. Then there are a strictly increasing sequence $\left(n_{i}\right)$ of natural numbers, a sequence $\left(V_{i}^{\prime}\right)$ of non-empty open subsets of $X$ and $x \in X$ such that

i) $V_{i}^{\prime} \subset V_{n_{i}}$ for every $i$,

ii) $\lim _{i \rightarrow \infty}\left|V_{i}^{\prime}\right|=\lim _{i \rightarrow \infty} \operatorname{dist}\left(x, V_{i}^{\prime}\right)=0$, and

iii) $\sum_{i} \operatorname{Cap}_{t}\left(V_{i}^{\prime}\right)=\infty$.

Proof. Let $B_{1}=\{x \in X ; d(x, B)<1\}$ and for each $n$, let $\mu_{n}$ be a probability measure on $V_{n}$ such that $I_{t}\left(\mu_{n}\right) \leq 2 \operatorname{Cap}_{t}\left(V_{n}\right)^{-1}$. Consider any $r \in(0,1)$ and let $A_{n}=\left\{x \in X ; \mu_{n}(B(x, r))>0\right\}$, and for $x \in A_{n}$ let $\mu_{n}^{x}=\left.\mu_{n}(B(x, r))^{-1} \mu_{n}\right|_{B(x, r)}$. Using Cauchy-Schwartz' inequality, Fubini's theorem and then that $B(y, r) \subset B_{1}$ for $\mu_{n}$-almost every $y$ for every $n$,

$$
\begin{aligned}
\int_{B_{1}} \mu_{n}(B(x, r))^{2} \mathrm{~d} \lambda(x) & \geq \frac{1}{\lambda\left(B_{1}\right)}\left(\int_{B_{1}} \mu_{n}(B(x, r)) \mathrm{d} \lambda(x)\right)^{2} \\
& =\frac{1}{\lambda\left(B_{1}\right)}\left(\int \lambda\left(B_{1} \cap B(y, r)\right) \mathrm{d} \mu_{n}(y)\right)^{2} \geq \frac{c^{-2} r^{2 d}}{\lambda\left(B_{1}\right)} .
\end{aligned}
$$

Thus for any natural number $a$,

$$
\begin{aligned}
\int_{B_{1}} \sum_{n=a}^{\infty} \operatorname{Cap}_{t}\left(V_{n} \cap B(x, r)\right) \mathrm{d} \lambda(x) & \geq \int_{B_{1}} \sum_{\substack{n \geq a \\
x \in A_{n}}} \frac{1}{I_{t}\left(\mu_{n}^{x}\right)} \mathrm{d} \lambda(x) \\
& \geq \sum_{n=a}^{\infty} \frac{1}{I_{t}\left(\mu_{n}\right)} \int_{B_{1} \cap A_{n}} \mu_{n}(B(x, r))^{2} \mathrm{~d} \lambda(x) \\
& =\sum_{n=a}^{\infty} \frac{1}{I_{t}\left(\mu_{n}\right)} \int_{B_{1}} \mu_{n}(B(x, r))^{2} \mathrm{~d} \lambda(x)=\infty .
\end{aligned}
$$

It follows that $\sum_{n=a}^{\infty} \operatorname{Cap}_{t}\left(V_{n} \cap B(x, r)\right)$ is unbounded as a function of $x$, and hence there exist $x \in B_{1}$ and a natural number $b$ such that

$$
\sum_{n=a}^{b} \operatorname{Cap}_{t}\left(V_{n} \cap B(x, r)\right) \geq 1 .
$$

It is now possible to recursively define a strictly increasing sequence $\left(m_{k}\right)$ of natural numbers and a sequence $\left(x_{k}\right)$ of points in $B_{1}$, such that $m_{1}=1$ and for 
every $k$,

$$
\sum_{n=m_{k}}^{m_{k+1}-1} \operatorname{Cap}_{t}\left(V_{n} \cap B\left(x_{k}, 2^{-k}\right)\right) \geq 1 .
$$

Let $\left(k_{j}\right)$ be a subsequence of the natural numbers such that $\left(x_{k_{j}}\right)$ converges to a point $x$ in $X$, and let $\left(n_{i}\right)$ be the increasing enumeration of the set

$$
\bigcup_{j}\left\{n ; m_{k_{j}} \leq n<m_{k_{j}+1} \text { and } V_{n} \cap B\left(x_{k_{j}}, 2^{-k_{j}}\right) \neq \emptyset\right\} .
$$

Then the sequence $\left(V_{i}^{\prime}\right)$ defined by

$$
V_{i}^{\prime}=V_{n_{i}} \cap B\left(x_{k_{j}}, 2^{-k_{j}}\right),
$$

where $k_{j}$ is such that $m_{k_{j}} \leq n_{i}<m_{k_{j}+1}$, has the properties $i$ )-iii).

LEMMA 3.4. Let $\left(b_{n}\right)$ be a sequence of positive numbers bounded away from 0 , such that $\sum_{n} b_{n}^{-1}=\infty$. Then there are non-negative numbers $\left(a_{n, k}\right)_{n, k \in \mathbb{N}}$ such that

i) $\left(a_{n, k}\right)_{k}$ has finite support for every $n$ and $\lim _{n \rightarrow \infty} \min \left\{k ; a_{n, k} \neq 0\right\}=$ $\infty$

ii) $\sum_{k} a_{n, k}=1$ for every $n$ and $\sum_{n, k} a_{n, k}^{2}<\infty$, and

iii) $\lim _{n \rightarrow \infty} \sum_{k} a_{n, k}^{2} b_{k}=0$.

Proof. Let

$$
a_{n, k}= \begin{cases}a_{n} b_{k}^{-1} & \text { if } M_{n} \leq k \leq N_{n} \\ 0 & \text { otherwise }\end{cases}
$$

where

$$
a_{n}=\frac{1}{\sum_{k=M_{n}}^{N_{n}} b_{k}^{-1}} .
$$

Since $\sum_{n} b_{n}^{-1}=\infty$ it is possible to choose $\left(M_{n}\right)$ and $\left(N_{n}\right)$ such that $\left.i\right)$ holds and $\sum_{n} a_{n}<\infty$. Then clearly $\sum_{k} a_{n, k}=1$ for every $n$, and

$$
\sum_{n, k} a_{n, k}^{2} \leq B \sum_{n}\left(a_{n} \sum_{k} a_{n, k}\right)=B \sum_{n} a_{n}<\infty,
$$

where $B=\sup _{k} b_{k}^{-1}$. Finally,

$$
\sum_{k} a_{n, k}^{2} b_{k}=\frac{\sum_{k=M_{n}}^{N_{n}} b_{k}^{-1}}{\left(\sum_{k=M_{n}}^{N_{n}} b_{k}^{-1}\right)^{2}}=a_{n}
$$

which converges to 0 when $n \rightarrow \infty$. 
Lemma 3.5. Let $\mu$ be a probability measure on a compact metric space $X$, and define the probability space $(\Omega, \mathbf{P})$ by $\Omega=X^{\mathbb{N}}$ and $\mathbf{P}=\mu^{\mathbb{N}}$. Let $\left(a_{n, k}\right)$ be non-negative numbers such that $\sum_{k} a_{n, k}=1$ for every $n$ and $\sum_{n, k} a_{n, k}^{2}<\infty$. For $\omega \in \Omega$, let

$$
\mu_{n}^{\omega}=\sum_{k} a_{n, k} \delta_{\omega_{k}} .
$$

Then almost surely $\lim _{n \rightarrow \infty} \mu_{n}^{\omega}=\mu$.

Proof. Let $\eta$ be a continuous function on $X$. Then for every $k$,

$$
\mathbf{E} \eta\left(\omega_{k}\right)=\mu(\eta)
$$

and

and hence

$$
\operatorname{Var} \eta\left(\omega_{k}\right)=\mu\left(\eta^{2}\right)-\mu(\eta)^{2} \leq\|\eta\|_{\infty}^{2}
$$

and

$$
\mathbf{E}\left(\mu_{n}^{\omega}(\eta)\right)=\mu(\eta)
$$

$$
\operatorname{Var}\left(\mu_{n}^{\omega}(\eta)\right)=\sum_{k} a_{n, k}^{2} \operatorname{Var} \eta\left(\omega_{k}\right) \leq\|\eta\|_{\infty}^{2} \sum_{k} a_{n, k}^{2} .
$$

Let $\varepsilon>0$. Then by Chebyshev's inequality,

$$
\sum_{n} \mathbf{P}\left\{\left|\mu_{n}^{\omega}(\eta)-\mu(\eta)\right| \geq \varepsilon\right\} \leq \sum_{n} \frac{\|\eta\|_{\infty}^{2} \sum_{k} a_{n, k}^{2}}{\varepsilon^{2}}<\infty,
$$

so that Borel-Cantelli's lemma implies that

$$
\limsup _{n \rightarrow \infty}\left|\mu_{n}^{\omega}(\eta)-\mu(\eta)\right| \leq \varepsilon
$$

almost surely. Since the space of continuous functions on $X$ is separable, it follows that $\lim _{n \rightarrow \infty} \mu_{n}^{\omega}=\mu$ almost surely.

LEMMA 3.6. Let $\left(\xi_{n}\right)$ be a sequence of independent random variables. Then almost surely

$$
\liminf _{n \rightarrow \infty} \xi_{n} \leq \liminf _{n \rightarrow \infty} \mathbf{E} \xi_{n} .
$$

Proof. By taking a subsequence it may be assumed that $\lim _{n \rightarrow \infty} \mathbf{E} \xi_{n}$ exists. Let $\left(\varepsilon_{n}\right)$ be a sequence of positive numbers converging to 0 , such that

$$
\sum_{n} \frac{\varepsilon_{n}}{1+\varepsilon_{n}}=\infty
$$

By Markov's inequality,

$\mathbf{P}\left(\xi_{n} \leq\left(1+\varepsilon_{n}\right) \mathbf{E} \xi_{n}\right)=1-\mathbf{P}\left(\xi_{n}>\left(1+\varepsilon_{n}\right) \mathbf{E} \xi_{n}\right) \geq 1-\frac{\mathbf{E} \xi_{n}}{\left(1+\varepsilon_{n}\right) \mathbf{E} \xi_{n}}=\frac{\varepsilon_{n}}{1+\varepsilon_{n}}$. 
Then by Borel-Cantelli's lemma there is almost surely a strictly increasing sequence $\left(n_{k}\right)$ of natural numbers such that $\xi_{n_{k}} \leq\left(1+\varepsilon_{n_{k}}\right) \mathbf{E} \xi_{n_{k}}$ for every $k$, and thus

$$
\liminf _{n \rightarrow \infty} \xi_{n} \leq \liminf _{k \rightarrow \infty} \xi_{n_{k}} \leq \liminf _{k \rightarrow \infty} \mathbf{E} \xi_{n_{k}} \leq \limsup _{n \rightarrow \infty} \mathbf{E} \xi_{n}=\liminf _{n \rightarrow \infty} \mathbf{E} \xi_{n} .
$$

Proof of Theorem 1.3. By Lemma 3.3 it may be assumed that $V_{n}$ is nonempty for every $n$ and

$$
\lim _{n \rightarrow \infty}\left|V_{n}\right|=\lim _{n \rightarrow \infty} \operatorname{dist}\left(g, V_{n}\right)=0
$$

for some $g \in G$.

Define a sequence $\left(\varphi_{n}^{\omega}\right)$ of random continuous functions on $G$ in the following way. For each $n$, there is a probability measure $\theta_{n}$ on $V_{n}$ such that $I_{t}\left(\theta_{n}\right) \leq 2 \operatorname{Cap}_{t}\left(V_{n}\right)^{-1}$, and an open subset $A_{n}$ of $V_{n}$ such that $d\left(A_{n}, V_{n}^{c}\right)>0$ and $\theta_{n}\left(A_{n}\right) \geq 1 / 2$. By Lemma 3.2 there is then a non-negative continuous function $\psi_{n}^{\prime}$ on $G$ such that $\left(\psi_{n}^{\prime} \mathrm{d} \lambda\right)\left(A_{n}\right) \geq \theta_{n}\left(A_{n}\right) / 2 \geq 1 / 4$ and $I_{t}\left(\psi_{n}^{\prime}\right) \leq 2 I_{t}\left(\theta_{n}\right) \leq$ $4 \operatorname{Cap}_{t}\left(V_{n}\right)^{-1}$. Let $\psi_{n}^{\prime \prime}$ be a continuous function on $G$ such that $\chi_{A_{n}} \leq \psi_{n}^{\prime \prime} \leq \chi_{V_{n}}$ and let $\psi_{n}=c_{n} \psi_{n}^{\prime} \psi_{n}^{\prime \prime}$, where $c_{n}$ is such that $\psi_{n} \mathrm{~d} \lambda$ is a probability measure. Then $c_{n} \leq 4$ and hence

$$
I_{t}\left(\psi_{n}\right) \leq c_{n}^{2} I_{t}\left(\psi_{n}^{\prime}\right) \leq 64 \operatorname{Cap}_{t}\left(V_{n}\right)^{-1},
$$

so that the hypothesis of the theorem implies that $\sum_{n} I_{t}\left(\psi_{n}\right)^{-1}=\infty$. Let $\left(a_{n, k}\right)$ be as in Lemma 3.4 with respect to $b_{n}=I_{t}\left(\psi_{n}\right)\left(\geq\left|V_{n}\right|^{-t}\right)$, and let

$$
\varphi_{n}^{\omega}=\sum_{k} a_{n, k} \psi_{k}^{\omega}
$$

where $\psi_{k}^{\omega}(x)=\psi_{k}\left(\omega_{k}^{-1} x\right)$.

Property ii) of Lemma 3.4 together with Lemma 3.5 applied in the space $\left(\bar{W},\left.\lambda\right|_{W}\right)$ and the right invariance of $\lambda$ imply that for almost every $\omega$,

$$
\lim _{n \rightarrow \infty} \sum_{k} a_{n, k} \delta_{\omega_{k} g}=\left.\lambda\right|_{W g} .
$$

Consider an arbitrary non-negative continuous function $\eta$ on $G$ such that supp $\eta \subset$ $W g$ and let $\nu=\eta \mathrm{d} \lambda$ (specific choices for $\eta$ will be made later). By (2) it follows for all $\omega$ satisfying (3) that $\lim _{n \rightarrow \infty} \varphi_{n}^{\omega} \mathrm{d} \lambda=\left.\lambda\right|_{W g}$ and thus

$$
\lim _{n \rightarrow \infty} \varphi_{n}^{\omega} \mathrm{d} \nu=\left.\eta \mathrm{d} \lambda\right|_{W g}=\nu .
$$


Let $\rho$ be a continuous function on $G$ with compact support. Using that $\psi_{i}^{\omega}(x)$ and $\psi_{j}^{\omega}(y)$ are independent for $i \neq j$,

$$
\begin{aligned}
\mathbf{E} I_{t}\left(\varphi_{n}^{\omega} \rho \mathrm{d} \nu\right) & =\sum_{k} a_{n, k}^{2} \mathbf{E} I_{t}\left(\psi_{k}^{\omega} \rho \mathrm{d} \nu\right) \\
& +\sum_{i \neq j} a_{n, i} a_{n, j} \iint d(x, y)^{-t} \mathbf{E} \psi_{i}^{\omega}(x) \mathbf{E} \psi_{j}^{\omega}(y) \rho(x) \rho(y) \mathrm{d} \nu(x) \mathrm{d} \nu(y) .
\end{aligned}
$$

Since $I_{t}\left(\psi_{k}^{\omega} \rho \mathrm{d} \nu\right) \leq\|\rho\|_{\infty}^{2}\|\eta\|_{\infty}^{2} I_{t}\left(\psi_{k}\right)$, it follows from property iii) of Lemma 3.4 that the first sum converges to 0 when $n \rightarrow \infty$. Next,

$$
\mathbf{E} \psi_{i}^{\omega}(x)=\left.\int \psi_{i}\left(\omega_{i}^{-1} x\right) \mathrm{d} \lambda\right|_{W}\left(\omega_{i}\right) \leq \int \psi_{i}\left(\omega_{i}^{-1} x\right) \mathrm{d} \lambda\left(\omega_{i}\right)=\lambda\left(\psi_{i}\right)=1,
$$

using that $\lambda$ is invariant under right translation and inversion. Thus the integral in the second sum is less than or equal to $I_{t}(\rho \mathrm{d} \nu)$ and it follows by property ii) in Lemma 3.4 that the second sum is less than or equal to $I_{t}(\rho \mathrm{d} \nu)$ as well. Thus by Lemma 3.6, almost surely

$$
\liminf _{n \rightarrow \infty} I_{t}\left(\varphi_{n}^{\omega} \rho \mathrm{d} \nu\right) \leq I_{t}(\rho \mathrm{d} \nu) .
$$

Lemma 1.4 applied in the space $(\bar{W} g, \nu)$ together with (1) and property i) of Lemma 3.4 now imply that almost surely

$$
\mathcal{H}^{t}(\operatorname{supp} \eta \cap E(\omega)) \geq \frac{\lambda(\eta)^{2}}{I_{t}(\eta)} .
$$

For $m=1,2, \ldots$, let $\mathcal{B}_{m}$ be a maximal collection of disjoint open balls in $W g$ of radius $2^{-m}$. For each $B \in \bigcup_{m} \mathcal{B}_{m}$, let $\eta_{B}$ be a non-negative continuous function on $G$ such that $\chi_{\frac{1}{2} B} \leq \eta_{B} \leq \chi_{B}$, where $\frac{1}{2} B$ is the ball concentric with $B$ having half the radius. Since $\bigcup_{m} \mathcal{B}_{m}$ is countable, almost every $\omega$ is such that whenever $B \in \mathcal{B}_{m}$ then

$$
\mathcal{H}^{t}(B \cap E(\omega)) \geq \frac{\lambda\left(\eta_{B}\right)^{2}}{I_{t}\left(\eta_{B}\right)} \geq C 2^{-t m},
$$

where the last inequality holds by Lemma 3.1 for some constant $C$ that is independent of $m$.

Let $\omega$ be such and let $\mathcal{U}$ be an open subset of $W g$. Since $(G, \lambda)$ is $d$-regular, there is a positive constant $C^{\prime}$ and some $m_{0}$ such that if $m \geq m_{0}$ then

$$
\#\left\{B \in \mathcal{B}_{m} ; B \subset \mathcal{U}\right\} \geq C^{\prime} 2^{d m} \text {. }
$$

Thus for $m \geq m_{0}$,

$$
\mathcal{H}^{t}(\mathcal{U} \cap E(\omega)) \geq \sum_{\substack{B \in \mathcal{B}_{m} \\ B \subset \mathcal{U}}} \mathcal{H}^{t}(B \cap E(\omega)) \geq C C^{\prime} 2^{(d-t) m},
$$


and letting $m \rightarrow \infty$ shows that $\mathcal{H}^{t}(\mathcal{U} \cap E(\omega))=\infty$.

\section{Hausdorff content and energy of rectangles in $\mathbb{H}$}

The purpose of this section is to estimate the Hausdorff content and energy of a rectangle $\bar{R}(x, r)$ in the Heisenberg group, up to multiplicative constants. Only upper bounds are provided, but it follows from (1) that they are the best possible ones. The multiplicative constants will mostly be implicit, using the following notation. If $e_{1}$ and $e_{2}$ are expressions depending on some parameters, then $e_{1} \lesssim e_{2}$ means that there is a positive constant $C$ such that $e_{1} \leq C e_{2}$ for all parameter values. Often some of the parameters in $e_{1}$ and $e_{2}$ will be considered as constants - then $C$ may depend on those parameters. For example, the implicit constants always depend on $t$.

4.1. Upper bound for the Hausdorff content of a rectangle

LEMmA 4.1. For all $t \in[0,4]$,

$$
\mathcal{H}_{\infty}^{t}(\bar{R}(0, r)) \lesssim \Phi^{t}(r)
$$

where the implicit constant depends on $t$ but not on $r$.

Proof. Let $R=\bar{R}(0, r)$. It is enough to show that if $r_{1} \leq r_{2}$, then

$$
\mathcal{H}_{\infty}^{t}(R) \lesssim \begin{cases}r_{2}^{t} & \text { if } t \in[0,2] \\ r_{1}^{t-2} r_{2}^{2} & \text { if } t \in[2,4]\end{cases}
$$

and if $r_{1} \geq r_{2}$, then

$$
\mathcal{H}_{\infty}^{t}(R) \lesssim \begin{cases}r_{1}^{t} & \text { if } t \in[0,3] \\ r_{1}^{6-t} r_{2}^{2(t-3)} & \text { if } t \in[3,4]\end{cases}
$$

For $t \geq 0$

$$
\mathcal{H}_{\infty}^{t}(R) \leq|R|^{t} \lesssim \max \left(r_{1}^{t}, r_{2}^{t}\right)= \begin{cases}r_{2}^{t} & \text { if } r_{1} \leq r_{2} \\ r_{1}^{t} & \text { if } r_{1} \geq r_{2}\end{cases}
$$

The vertical segment $S=\{0\} \times\left[-r_{2}^{2}, r_{2}^{2}\right]$ is $r_{1}$-dense in $R$, and

$$
D=\left\{\left(0, k r_{1}^{2}\right) ; k=-\left\lfloor\frac{r_{2}^{2}}{r_{1}^{2}}\right\rfloor, \ldots,\left\lfloor\frac{r_{2}^{2}}{r_{1}^{2}}\right\rfloor\right\}
$$

is $r_{1}$-dense in $S$ - thus $D$ is $2 r_{1}$-dense in $R$. If $r_{1} \leq r_{2}$ then $\# D \lesssim r_{2}^{2} / r_{1}^{2}$, and hence

$$
\mathcal{H}_{\infty}^{t}(R) \lesssim \frac{r_{2}^{2}}{r_{1}^{2}} \cdot r_{1}^{t}=r_{1}^{t-2} r_{2}^{2}
$$


It remains to show that $\mathcal{H}_{\infty}^{t}(R) \lesssim r_{1}^{6-t} r_{2}^{2(t-3)}$ for $r_{1} \geq r_{2}$ and $t \in[3,4]$. This is done by estimating the Hausdorff content of annuli of the form

$$
A_{\rho}=\left\{(x, y, z) ;\left|\left(x^{2}+y^{2}\right)^{1 / 2}-\rho\right| \leq \frac{r_{2}^{2}}{2 \rho},|z| \leq r_{2}^{2}\right\} .
$$

Let

$$
C_{\rho}=\left\{(x, y, 0) ; x^{2}+y^{2}=\rho^{2}\right\} .
$$

Sublemma. For $\rho \geq r_{2}$, the set $C_{\rho}$ is $\sqrt{2} r_{2}^{2} / \rho$-dense in $A_{\rho}$.

Proof. Let $p=(\rho, 0,0)$ and let

$$
S=\{(x, y, z) ; x \geq 0, z=2 \rho y\}
$$

this is the part of the plane $H(p)$ defined in the introduction where the $x$ coordinate is non-negative. Let $q=(x, y, z)$ be a point in $A_{\rho} \cap S$. Then

and

$$
x \leq \rho+\frac{r_{2}^{2}}{2 \rho} \quad \text { and } \quad|y|=\frac{|z|}{2 \rho} \leq \frac{r_{2}^{2}}{2 \rho},
$$

so that

$$
x^{2}+y^{2} \geq\left(\rho-\frac{r_{2}^{2}}{2 \rho}\right)^{2}
$$

$$
\begin{aligned}
x \geq\left(\left(\rho-\frac{r_{2}^{2}}{2 \rho}\right)^{2}-y^{2}\right)^{1 / 2} & \geq\left(\left(\rho-\frac{r_{2}^{2}}{2 \rho}\right)^{2}-\left(\frac{r_{2}^{2}}{2 \rho}\right)^{2}\right)^{1 / 2} \\
& =\left(\rho^{2}-r_{2}^{2}\right)^{1 / 2} \geq \rho-\frac{r_{2}^{2}}{\rho}
\end{aligned}
$$

(the last inequality is proved by squaring both sides and using that $\rho \geq r_{2}$ ). Thus

$$
d_{\mathbb{H}}(p, q)=\left((x-\rho)^{2}+y^{2}\right)^{1 / 2} \leq \sqrt{2} \max (|x-\rho|,|y|) \leq \frac{\sqrt{2} r_{2}^{2}}{\rho} .
$$

Let $R_{\alpha}$ be the rotation by $\alpha$ around the vertical axis $\{0\} \times \mathbb{R}$. The statement follows since $d_{\mathbb{H}}$ is invariant under $R_{\alpha}$ and

$$
C_{\rho}=\bigcup_{\alpha} R_{\alpha}(p) \quad \text { and } \quad A_{\rho}=\bigcup_{\alpha} R_{\alpha}\left(A_{\rho} \cap S\right) .
$$

Sublemma. Let $\varepsilon>0$. The set

$$
D_{\rho}=\left\{\left(\rho \cos \left(\frac{k \varepsilon^{2}}{2 \rho^{2}}\right), \rho \sin \left(\frac{k \varepsilon^{2}}{2 \rho^{2}}\right), 0\right) ; k=0, \ldots,\left\lfloor\frac{4 \pi \rho^{2}}{\varepsilon^{2}}\right\rfloor\right\}
$$

is $\varepsilon$-dense in $C_{\rho}$. 
Proof. The points $p=(\rho, 0,0)$ and $q=(\rho \cos \alpha, \rho \sin \alpha, 0)$ satisfy

$$
\begin{aligned}
d_{\mathbb{H}}(p, q) & =\rho\left(\left((1-\cos \alpha)^{2}+\sin ^{2} \alpha\right)^{2}+(2 \sin \alpha)^{2}\right)^{1 / 4} \\
& =2^{3 / 4} \rho(1-\cos \alpha)^{1 / 4} \leq \rho|2 \alpha|^{1 / 2},
\end{aligned}
$$

using that $\cos \alpha \geq 1-\alpha^{2} / 2$. The same bound holds for any pair of points $p, q$ on $C_{\rho}$ making an angle $\alpha$, and taking $\alpha=\varepsilon^{2} / 2 \rho^{2}$ gives $d_{\mathbb{H}}(p, q) \leq \varepsilon$.

Proof of Lemma 4.1 (COntinued). Take $\varepsilon=r_{2}^{2} / \rho$ in the definition of $D_{\rho}$. Then for $\rho \geq r_{2}$ the set $D_{\rho}$ is $3 r_{2}^{2} / \rho$-dense in $A_{\rho}$ and $\# D_{\rho} \lesssim \rho^{4} / r_{2}^{4}$, and thus

$$
\mathcal{H}_{\infty}^{t}\left(A_{\rho}\right) \lesssim \frac{\rho^{4}}{r_{2}^{4}} \cdot\left(\frac{r_{2}^{2}}{\rho}\right)^{t}=r_{2}^{2 t-4} \rho^{4-t} .
$$

Let $\rho_{k}=r_{2} \sqrt{k}$. Then

$$
\rho_{k+1}-\rho_{k}=r_{2} \int_{k}^{k+1} \frac{1}{2 \sqrt{u}} \mathrm{~d} u \leq \frac{r_{2}}{2 \sqrt{k}}=\frac{r_{2}^{2}}{2 \rho_{k}},
$$

so that $A_{\rho_{k}}$ and $A_{\rho_{k+1}}$ overlap. Thus

$$
R \subset \bar{R}\left(0,\left(r_{2}, r_{2}\right)\right) \cup \bigcup_{k=1}^{\left\lceil\left(\frac{r_{1}}{r_{2}}\right)^{2}\right\rceil} A_{\rho_{k}} .
$$

It follows that

$$
\begin{aligned}
\mathcal{H}_{\infty}^{t}(R) & \lesssim r_{2}^{t}+\sum_{k=1}^{\left\lceil\left(\frac{r_{1}}{r_{2}}\right)^{2}\right\rceil} r_{2}^{2 t-4} \rho_{k}^{4-t}=r_{2}^{t}\left(1+\sum_{k=1}^{\left\lceil\left(\frac{r_{1}}{r_{2}}\right)^{2}\right\rceil} k^{(4-t) / 2}\right) \\
& \lesssim r_{2}^{t}\left(1+\left(\frac{r_{1}}{r_{2}}\right)^{6-t}\right) \lesssim r_{1}^{6-t} r_{2}^{2(t-3)},
\end{aligned}
$$

using in the last step that $\left(r_{1} / r_{2}\right)^{6-t} \geq 1$. This completes the proof of Lemma 4.1.

4.2. Upper bound for the energy of a rectangle

LEMma 4.2. For all $t \in(0,4) \backslash\{1,2,3\}$,

$$
\Phi^{t}(r) \lesssim \operatorname{Cap}_{t}(\bar{R}(x, r)),
$$

where the implicit constant depends on $t$ but not on $r$. 
Proof. Let $R=\bar{R}(0, r)$. Since

$$
\operatorname{Cap}_{t}(R) \geq \frac{\lambda(R)^{2}}{I_{t}(R)}
$$

it is enough to show that if $r_{1} \leq r_{2}$, then

$$
I_{t}(R) \lesssim \begin{cases}r_{1}^{4} r_{2}^{4-t} & \text { if } t \in(0,2) \\ r_{1}^{6-t} r_{2}^{2} & \text { if } t \in(2,4)\end{cases}
$$

and if $r_{1} \geq r_{2}$, then

$$
I_{t}(R) \lesssim \begin{cases}r_{1}^{4-t} r_{2}^{4} & \text { if } t \in(0,3) \backslash\{1\} \\ r_{1}^{t-2} r_{2}^{2(5-t)} & \text { if } t \in(3,4)\end{cases}
$$

Let

$$
R_{t}(p)=\int_{R} d_{\mathbb{H}}(p, q)^{-t} \mathrm{~d} \lambda(q),
$$

so that

$$
I_{t}(R)=\int_{R} R_{t}(p) \mathrm{d} \lambda(p) .
$$

Since $R, d_{\mathbb{H}}$ and $\lambda$ are invariant under rotation around the vertical axis, the integral defining $R_{t}(p)$ does not depend on the angle of $p$ in the horizontal plane. To estimate $R_{t}(p)$ it is therefore enough to consider $p$ of the form $p=\left(\rho, 0, z_{0}\right)$. Assume that $\rho \in\left[0, r_{1}\right]$ and $z_{0} \in\left[-r_{2}^{2}, r_{2}^{2}\right]$, so that $p \in R$.

Let

$$
f_{\rho}(x, y, z)=\max \left(|x|,|y|,|z-2 \rho y|^{1 / 2}\right) .
$$

Then for $q=(x, y, z)$,

$$
d_{\mathbb{H}}(p, q)=\left(\left((x-\rho)^{2}+y^{2}\right)^{2}+\left(z-z_{0}-2 \rho y\right)^{2}\right)^{1 / 4} \approx f_{\rho}\left(x-\rho, y, z-z_{0}\right) .
$$

Define the Euclidean rectangles

$$
\begin{aligned}
& A=\left[-2 r_{1}, 2 r_{1}\right] \times\left[-2 r_{1}, 2 r_{1}\right] \times\left[-2 r_{2}^{2}, 2 r_{2}^{2}\right], \\
& A^{\prime}=\left(\rho, 0, z_{0}\right)+A,
\end{aligned}
$$


where + means Euclidean translation, and let $B(a)=\left\{q ; f_{\rho}(q) \leq a\right\}$. Since $R \subset A^{\prime}$,

$$
\begin{aligned}
R_{t}(p) & \leq \int_{A^{\prime}} d_{\mathbb{H}}(p, q)^{-t} \mathrm{~d} \lambda(q) \lesssim \int_{A^{\prime}} f_{\rho}\left(x-\rho, y, z-z_{0}\right)^{-t} \mathrm{~d} \lambda(x, y, z) \\
& =\int_{A} f_{\rho}(x, y, z)^{-t} \mathrm{~d} \lambda(x, y, z)=\int_{0}^{\infty} \lambda\left\{q \in A ; f_{\rho}(q)^{-t} \geq \gamma\right\} \mathrm{d} \gamma \\
& =\int_{0}^{\infty} \lambda\left(A \cap B\left(\gamma^{-1 / t}\right)\right) \mathrm{d} \gamma \approx \int_{0}^{\infty} \lambda(A \cap B(a)) a^{-(t+1)} \mathrm{d} a .
\end{aligned}
$$

To estimate $R_{t}(p)$ it is useful to have an upper bound for $\lambda(A \cap B(a))$.

The set $B(a)$ is the intersection of the vertical cylinder $[-a, a] \times[-a, a] \times \mathbb{R}$ with the set of points having vertical Euclidean distance at most $a^{2}$ to the plane $z=2 \rho y$. In particular, the projection of $B(a)$ to the $y z$-plane is the intersection of the strips

$$
S_{1}=\{(y, z) ;-a \leq y \leq a\}, \quad S_{2}=\left\{(y, z) ; 2 \rho y-a^{2} \leq z \leq 2 \rho y+a^{2}\right\} .
$$

The projection of $A$ to the $y z$-plane is the intersection of the strips

$$
S_{3}=\left\{(y, z) ;-2 r_{1} \leq y \leq 2 r_{1}\right\}, \quad S_{4}=\left\{(y, z) ;-2 r_{2}^{2} \leq z \leq 2 r_{2}^{2}\right\} .
$$

It is used in the computations below that if $u \neq-1$ and $0 \leq v \leq w \leq \infty$ then

$$
\int_{v}^{w} a^{u} \mathrm{~d} a \lesssim \max \left(v^{u+1}, w^{u+1}\right),
$$

with the convention that $1 / 0=\infty$, and where the implicit constant depends on $u$ but not on $v, w$.

Sublemma. It holds that $\lambda(A \cap B(a)) \lesssim \min \left(a^{4}, r_{1}^{2} a^{2}, r_{1}^{2} r_{2}^{2}\right)$.

Proof. The projection of $A \cap B(a)$ to the $y z$-plane is contained in each of the parallelograms

$$
S_{1} \cap S_{2}, \quad S_{3} \cap S_{4}, \quad S_{2} \cap S_{3} .
$$

The first two have area $4 a^{3}$ and $16 r_{1} r_{2}^{2}$, respectively, and the third has vertices

$$
\left(-2 r_{1},-4 \rho r_{1} \pm a^{2}\right), \quad\left(2 r_{1}, 4 \rho r_{1} \pm a^{2}\right),
$$

and hence area $8 r_{1} a^{2}$. The extension of $A$ in the $x$-direction is $4 r_{1}$ and the extension of $B(a)$ in the $x$-direction is $2 a$. Thus

$$
\lambda(A \cap B(a)) \lesssim \min \left(a^{3}, r_{1} r_{2}^{2}, r_{1} a^{2}\right) \cdot \min \left(r_{1}, a\right) \leq \min \left(a^{4}, r_{1}^{2} a^{2}, r_{1}^{2} r_{2}^{2}\right) .
$$


Proof of Lemma 4.2 (Continued). The Case $r_{1} \leq r_{2}$. Let $t \in(0,4) \backslash$ $\{2\}$. Using (4), the sublemma and (5),

$$
\begin{aligned}
R_{t}(p) & \lesssim \int_{0}^{\infty} \min \left(a^{4}, r_{1}^{2} a^{2}, r_{1}^{2} r_{2}^{2}\right) a^{-(t+1)} \mathrm{d} a \\
& \leq \int_{0}^{r_{1}} a^{3-t} \mathrm{~d} a+\int_{r_{1}}^{r_{2}} r_{1}^{2} a^{1-t} \mathrm{~d} a+\int_{r_{2}}^{\infty} r_{1}^{2} r_{2}^{2} a^{-(t+1)} \mathrm{d} a \\
& \lesssim \max \left(r_{1}^{4-t}, r_{1}^{2} r_{2}^{2-t}\right) .
\end{aligned}
$$

It follows that

$I_{t}(R) \lesssim \lambda(R) \cdot \max \left(r_{1}^{4-t}, r_{1}^{2} r_{2}^{2-t}\right)=\max \left(r_{1}^{6-t} r_{2}^{2}, r_{1}^{4} r_{2}^{4-t}\right)= \begin{cases}r_{1}^{4} r_{2}^{4-t} & \text { if } t \in(0,2), \\ r_{1}^{6-t} r_{2}^{2} & \text { if } t \in(2,4)\end{cases}$

Sublemma. It holds that $\lambda(A \cap B(a)) \lesssim \min \left(a^{4}, r_{2}^{2} a^{3} / \rho, r_{1} r_{2}^{2} a, r_{1}^{2} r_{2}^{2}\right)$.

Proof. The projection of $A \cap B(a)$ to the $y z$-plane is contained in each of the parallelograms

$$
S_{1} \cap S_{2}, \quad S_{3} \cap S_{4}, \quad S_{2} \cap S_{4}
$$

The first two have area $4 a^{3}$ and $16 r_{1} r_{2}^{2}$, respectively, and the third has vertices

$$
\left(\frac{-2 r_{2}^{2} \pm a^{2}}{2 \rho},-2 r_{2}^{2}\right), \quad\left(\frac{2 r_{2}^{2} \pm a^{2}}{2 \rho}, 2 r_{2}^{2}\right)
$$

and hence area $4 r_{2}^{2} a^{2} / \rho$. The extension of $A$ in the $x$-direction is $4 r_{1}$ and the extension of $B(a)$ in the $x$-direction is $2 a$. Thus

$$
\begin{aligned}
\lambda(A \cap B(a)) & \lesssim \min \left(a^{3}, r_{1} r_{2}^{2}, \frac{r_{2}^{2} a^{2}}{\rho}\right) \cdot \min \left(r_{1}, a\right) \\
& \leq \min \left(a^{4}, \frac{r_{2}^{2} a^{3}}{\rho}, r_{1} r_{2}^{2} a, r_{1}^{2} r_{2}^{2}\right) .
\end{aligned}
$$

Proof of Lemma 4.2 (Continued). The CASE $r_{1} \geq r_{2}$. Again, $R_{t}(p)$ can be estimated using (4), the sublemma and (5), but the estimate now depends on $\rho \in\left[0, r_{1}\right]$, the first component of the point $p$. Let $t \in(0,4) \backslash\{1,3\}$. Then for 
every $\rho$,

$$
\begin{aligned}
R_{t}(p) & \lesssim \int_{0}^{\infty} \min \left(a^{4}, r_{1} r_{2}^{2} a, r_{1}^{2} r_{2}^{2}\right) a^{-(t+1)} \mathrm{d} a \\
& \leq \int_{0}^{\left(r_{1} r_{2}^{2}\right)^{1 / 3}} a^{3-t} \mathrm{~d} a+\int_{\left(r_{1} r_{2}^{2}\right)^{1 / 3}}^{r_{1}} r_{1} r_{2}^{2} a^{-t} \mathrm{~d} a+\int_{r_{1}}^{\infty} r_{1}^{2} r_{2}^{2} a^{-(t+1)} \mathrm{d} a \\
& \lesssim \max \left(\left(r_{1} r_{2}^{2}\right)^{(4-t) / 3}, r_{1}^{2-t} r_{2}^{2}\right)= \begin{cases}r_{1}^{2-t} r_{2}^{2} & \text { if } t \in(0,1), \\
\left(r_{1} r_{2}^{2}\right)^{(4-t) / 3} & \text { if } t \in(1,4) \backslash\{3\} .\end{cases}
\end{aligned}
$$

For $t \in(1,4) \backslash\{3\}$ and $\rho \geq\left(r_{2}^{4} / r_{1}\right)^{1 / 3}$, the estimate can be made sharper. For such $\rho$, the interval $\left[r_{2}^{2} / \rho, \sqrt{\rho r_{1}}\right]$ is non-empty, and when $a$ lies in this interval the minimum in the expression given by the sublemma is achieved by the second option. Thus

$$
\begin{aligned}
R_{t}(p) & \lesssim \int_{0}^{\infty} \min \left(a^{4}, \frac{r_{2}^{2} a^{3}}{\rho}, r_{1} r_{2}^{2} a, r_{1}^{2} r_{2}^{2}\right) a^{-(t+1)} \mathrm{d} a \\
& \leq \int_{0}^{r_{2}^{2} / \rho} a^{3-t} \mathrm{~d} a+\int_{r_{2}^{2} / \rho}^{\sqrt{\rho r_{1}}} \frac{r_{2}^{2} a^{2-t}}{\rho} \mathrm{d} a+\int_{\sqrt{\rho r_{1}}}^{r_{1}} r_{1} r_{2}^{2} a^{-t} \mathrm{~d} a+\int_{r_{1}}^{\infty} r_{1}^{2} r_{2}^{2} a^{-(t+1)} \mathrm{d} a \\
& \lesssim \max \left(r_{2}^{2(4-t)} \rho^{t-4}, r_{1}^{(3-t) / 2} r_{2}^{2} \rho^{(1-t) / 2}, r_{1}^{2-t} r_{2}^{2}\right) \\
& \stackrel{*}{=} \begin{cases}r_{1}^{(3-t) / 2} r_{2}^{2} \rho^{(1-t) / 2} & \text { if } t \in(1,3), \\
r_{2}^{2(4-t)} \rho^{t-4} & \text { if } t \in(3,4) .\end{cases}
\end{aligned}
$$

Denoting the options in the maximum by $\mathrm{O}_{1}, \mathrm{O}_{2}, \mathrm{O}_{3}$,

$$
\frac{O_{1}^{2}}{O_{2}^{2}}=\left(\frac{r_{1} \rho^{3}}{r_{2}^{4}}\right)^{t-3}, \quad \frac{O_{2}^{2}}{O_{3}^{2}}=\left(\frac{r_{1}}{\rho}\right)^{t-1},
$$

and the equality $*$ follows using that the expressions in parentheses are greater than or equal to 1 .

Let $g_{t}(\rho)=\sup _{z_{0}} R_{t}(p)$ where $p=\left(\rho, 0, z_{0}\right)$. Then

$$
I_{t}(R) \lesssim r_{2}^{2} \int_{0}^{r_{1}} g_{t}(\rho) \rho \mathrm{d} \rho .
$$

For $t \in(0,1)$, the estimate (6) gives

$$
I_{t}(R) \lesssim r_{2}^{2} \int_{0}^{r_{1}} r_{1}^{2-t} r_{2}^{2} \rho \mathrm{d} \rho \approx r_{1}^{4-t} r_{2}^{4}
$$


For $t \in(1,4) \backslash\{3\}$, the first part of the integral $\int_{0}^{r_{1}} g_{t}(\rho) \rho \mathrm{d} \rho$ is again estimated using (6). Let

$$
I_{0}=r_{2}^{2} \int_{0}^{\left(r_{2}^{4} / r_{1}\right)^{1 / 3}}\left(r_{1} r_{2}^{2}\right)^{(4-t) / 3} \rho \mathrm{d} \rho \approx r_{1}^{(2-t) / 3} r_{2}^{2(11-t) / 3} .
$$

Then for $t \in(1,3)$,

$$
I_{t}(R) \lesssim I_{0}+r_{2}^{2} \int_{0}^{r_{1}} r_{1}^{(3-t) / 2} r_{2}^{2} \rho^{(3-t) / 2} \mathrm{~d} \rho \approx I_{0}+r_{1}^{4-t} r_{2}^{4} \approx r_{1}^{4-t} r_{2}^{4},
$$

using that $I_{0} \approx r_{1}^{(2-t) / 3} r_{2}^{2(5-t) / 3} r_{2}^{4} \leq r_{1}^{4-t} r_{2}^{4}$. For $t \in(3,4)$,

$$
I_{t}(R) \lesssim I_{0}+r_{2}^{2} \int_{0}^{r_{1}} r_{2}^{2(4-t)} \rho^{t-3} \mathrm{~d} \rho \approx I_{0}+r_{1}^{t-2} r_{2}^{2(5-t)} \approx r_{1}^{t-2} r_{2}^{2(5-t)},
$$

using that $I_{0} \approx r_{1}^{(2-t) / 3} r_{2}^{4(t-2) / 3} r_{2}^{2(5-t)} \leq r_{1}^{t-2} r_{2}^{2(5-t)}$. This completes the proof of Lemma 4.2 .

Combining Lemmas 4.1 and 4.2 proves Proposition 1.2.

\section{Proof of Theorem 1.1}

It is clear that $\operatorname{dim}_{\mathrm{H}} E_{\underline{r}}(\omega) \leq \operatorname{dim}_{\mathrm{H}} \mathbb{H}=4$. Let $t \in(0,4) \backslash\{1,2,3\}$.

For every $\omega$ and $n_{0}$,

$$
E_{\underline{r}}(\omega) \subset \bigcup_{n=n_{0}}^{\infty} \bar{R}\left(\omega_{n}, r_{n}\right),
$$

and by Lemma $4.1, \mathcal{H}_{\infty}^{t}\left(\bar{R}\left(\omega_{n}, r_{n}\right)\right) \lesssim \Phi^{t}\left(r_{n}\right)$. Thus for every $n_{0}$

$$
\mathcal{H}_{\infty}^{t}\left(E_{\underline{r}}(\omega)\right) \lesssim \sum_{n=n_{0}}^{\infty} \Phi^{t}\left(r_{n}\right)
$$

It follows that if $\sum_{n} \Phi^{t}\left(r_{n}\right)<\infty$ then $\mathcal{H}_{\infty}^{t}\left(E_{\underline{r}}(\omega)\right)=0$ so that $\operatorname{dim}_{\mathrm{H}} E_{\underline{r}}(\omega) \leq t$.

By Lemma 4.2, $\operatorname{Cap}_{t}\left(\bar{R}\left(0, r_{n}\right)\right) \gtrsim \Phi^{t}\left(r_{n}\right)$. Thus if $\sum_{n} \Phi^{t}\left(r_{n}\right)=\infty$ then Theorem 1.3 with $\lambda$ replaced by $\lambda(W)^{-1} \lambda$ implies that $\operatorname{dim}_{\mathrm{H}} E_{\underline{r}}(\omega) \geq t$ for almost every $\omega$.

ACKnowledgements. We gratefully acknowledge the support of the Centre of Excellence in Analysis and Dynamics Research, funded by the Academy of Finland, and the hospitality of Institut Mittag-Leffler where part of this work was carried out. We also thank De-Jun Feng and the referee for useful comments. 


\section{REFERENCES}

1. Balogh, Z., Berger, R., Monti, R., and Tyson, J., Exceptional sets for self-similar fractals in Carnot groups, Math. Proc. Cambridge Philos. Soc. 149 (2010), no. 1, 147-172.

2. Balogh, Z., Durand-Cartagena, E., Fässler, K., Mattila, P., and Tyson, J., The effect of projections on dimension in the Heisenberg group, Rev. Mat. Iberoam. 29 (2013), no. 2, 381-432.

3. Balogh, Z., Fässler, K., Mattila, P., and Tyson, J., Projection and slicing theorems in Heisenberg groups, Adv. Math. 231 (2012), no. 2, 569-604.

4. Balogh, Z., Rickly, M., and Serra Cassano, F., Comparison of Hausdorff measures with respect to the Euclidean and the Heisenberg metric, Publ. Mat. 47 (2003), no. 1, 237-259.

5. Balogh, Z., and Tyson, J., Hausdorff dimensions of self-similar and self-affine fractals in the Heisenberg group, Proc. London Math. Soc. (3) 91 (2005), no. 1, 153-183.

6. Balogh, Z., Tyson, J., and Warhurst, B., Sub-Riemannian vs. Euclidean dimension comparison and fractal geometry on Carnot groups, Adv. Math. 220 (2009), no. 2, 560-619.

7. Balogh, Z., Tyson, J., and Wildrick, K., Frequency of Sobolev dimension distortion of horizontal subgroups in Heisenberg groups, Ann. Sc. Norm. Super. Pisa Cl. Sci. (5) 17 (2017), no. 2, 655-683.

8. Besicovitch, A., On the sum of digits of real numbers represented in the dyadic system, Ann. Math. 110 (1935), no. 1, 321-330.

9. Borel, E., Sur les séries de Taylor, Acta Math. 21 (1897), no. 1, 243-247.

10. Cantelli, F., Sulla probabilitá come limite della frequenza, Atti Accad. Naz. Lincei 26 (1917), no. 1, 39-45.

11. Chousionis, V., Tyson, J., and Urbański, M., Conformal graph directed Markov systems on Carnot groups, Mem. Amer. Math. Soc., to appear, available in arXiv:1605.01127.

12. Durand, A., On randomly placed arcs on the circle, in "Recent developments in fractals and related fields", Appl. Numer. Harmon. Anal., Birkhäuser Boston Inc., 2010, pp. 343-351.

13. Eggleston, H., The fractional dimension of a set defined by decimal properties, Quart. J. Math. Oxford 20 (1949), 31-36.

14. Ekström, F., Järvenpää, E., Järvenpää, M., and Suomala, V., Hausdorff dimension of limsup sets of random rectangles in products of regular spaces, Proc. Amer. Math. Soc. 146 (2018), no. 6, 2509-2521.

15. Ekström, F., and Persson, T., Hausdorff dimension of random limsup sets, J. London Math. Soc. 98 (2018), no. 2, 661-686

16. Falconer, K., Sets with large intersection properties, J. London Math. Soc. (2) 49 (1994), no. 2, 267-280.

17. Fan, A.-H., Schmeling, J., and Troubetzkoy, S., A multifractal mass transference principle for Gibbs measures with applications to dynamical Diophantine approximation, Proc. Lond. Math. Soc. 107 (2013), no. 5, 1173-1219.

18. Fan, A.-H., and $\mathrm{Wu}$, J., On the covering by small random intervals, Ann. Inst. Henri Poincaré Probab. Stat. 40 (2004), no. 1, 125-131.

19. Fässler, K., and Hovila, R., Improved Hausdorff dimension estimate for vertical projections in the Heisenberg group, Ann. Sc. Norm. Super. Pisa Cl. Sci. (5) 15 (2016), no. 1, 459-483.

20. Feng, D.-J., Järvenpää, E., Järvenpää, M., and Suomala, V., Dimensions of random covering sets in Riemann manifolds, Ann. Probab. 46 (2018), no. 3, 1542-1596.

21. Hovila, R., Transversality of isotropic projections, unrectifiability, and Heisenberg groups, Rev. Mat. Iberoam. 30 (2014), no. 2, 463-476. 
22. Jarník, V., Zur theorie der diophantischen approximationen, Monatsh. Math. Phys. 39 (1932), no. 1, 403-438.

23. Järvenpää, E., Järvenpää, M., Koivusalo, H., Li, B., and Suomala, V., Hausdorff dimension of affine random covering sets in torus, Ann. Inst. Henri Poincaré Probab. Stat. 50 (2014), no. 4, 1371-1384.

24. Järvenpää, E., Järvenpää, M., Koivusalo, H., Li, B., Suomala, V., and Xiao, Y., Hitting probabilities of random covering sets in tori and metric spaces, Electron. J. Probab. 22 (2017), no. 1, 1-18.

25. Khintchine, A., Zur metrischen theorie der diophantischen approximationen, Math. Z. 24 (1926), no. 1, 706-714

26. Persson, T., A note on random coverings of tori, Bull. Lond. Math. Soc. 47 (2015), no. 1, $7-12$.

27. Persson, T., Inhomogeneous potentials, Hausdorff dimension and shrinking targets, Ann. Henri Lebesgue, to appear.

28. Persson, T., and Reeve, H., A Frostman-type lemma for sets with large intersections, and an application to Diophantine approximation, Proc. Edinb. Math. Soc. (2) 58 (2015), no. $2,521-542$.

29. Seuret, S., Inhomogeneous random coverings of topological Markov shifts, Math. Proc. Cambridge Philos. Soc. 165 (2018), no. 2, 341-357.

30. Seuret, S., and Vigneron, F., Multifractal analysis of functions on Heisenberg and Carnot groups, J. Inst. Math. Jussieu 16 (2017), no. 1, 1-38.

31. Vandehey, J., Diophantine properties of continued fractions on the Heisenberg group, Int. J. Number Theory 12 (2016), no. 2, 541-560.

32. Zheng, C., A shrinking target problem with target at infinity in rank one homogeneous spaces, arXiv:1610.01870v3 (2016).

Department of Mathematical SciENCES, P.O. Box 3000, 90014 University E-mail: esa.jarvenpaa@oulu.fi ${ }^{2}$ OF Oulu, Finland ${ }^{1,2,3}$

E-mail: j.fredrik.e@gmail.com ${ }^{1}$

E-mail: maarit.jarvenpaa@oulu.fi ${ }^{3}$ 